\title{
Role of breast cancer inhibitors on diabetes mellitus- an in silico approach
}

\author{
Shailima DV Rampogu
}

\begin{abstract}
Background: Breast cancer and diabetes mellitus type-2 are two of the major diseases, which cause death to the women worldwide. Around $16 \%$ of the breast cancer patients also suffer from diabetes mellitus. It has been noted that the risk of occurrence of the breast cancer is more in patients suffering from diabetes mellitus.

Methods: Owing to this, the present article deals with an objective of using the breast cancer inhibitors for the diabetes drug target- 1ADS. Ten breast cancer inhibitors were drawn using Marvin Sketch. The docking was performed using Molegro software (Molegro Virtual Docker, MVD).

Results: The docking wizard generated 50 poses with the highest Mol Dock score -131.649.

Conclusion: This investigation successfully evaluated the effect of breast cancer inhibitors on diabetes mellitus, providing a new dimension in endocrine research.
\end{abstract}

Keywords: Cancer, Diabetes mellitus, 1ADS, Molegro, MVD, Breast cancer inhibitors

\section{Introduction}

Today, cancer and diabetes are two of the major global health risks $[1,2]$. There are several reports delineating how diabetes mellitus triggers a variety of cancers [3-8]. By the year 2007, in the United Sates alone, it was noted that about $8 \%$ of the total adult population were diabetic and 2.5 million people were reported having breast cancer $[9,10]$. Therefore, it is important to probe the relationship between diabetes mellitus and breast cancer The epidemiological studies have reported that the insulin-like growth factor 1 evokes the growth of colon tumour, suggesting the tumour growth is associated with the levels of insulin [11-15]. Knowing the fact that diabetes mellitus provokes the tumour cells, it would be ideal to design drugs that can act on both the diseases. One of such drugs is Metformin [2], which is a known diabetic agent acts by reducing the increased levels of insulin in blood [2]. Metformin decreases hyperglycaemia and hyperinsulinemia by increasing skeletal muscle glucose uptake [2]. Increased levels of insulin-like growth factor 1 (IGF-1) in serum/plasma are seen in prostate cancer and pre menopausal breast cancer [16-20].

Correspondence: shailima.rampogu@gmail.com

Department of Biochemistry, Cachet Labs, Hyderabad, Telangana, India
The association of diabetes and breast cancer is a long debated topic. Among the cancers present today, breast cancer is considered to be one of the causes of deaths seen in women [21]. It was noted in $16 \%$ of the patients who suffer from breast cancer were found to be diagnosed with diabetes [22]. Diabetes brings about several modifications in hormonal system [22] which could trigger the enhancement of breast cancer like growth factors estrogen [22] and other growth factors. Diabetes inhibits the AMP kinase by decreasing the adiponectin plasma levels, which activates certain pathways like Akt and ERK aggravating the breast cancer risk Figure 1 [23,24].

Breast cancer is seen in association with type 2 diabetes in women who have reached their post menopausal stage. In most of the cases it was observed that the overweight/obese women are more likely to develop both diseases as depicted in Figure 2.

1 ADS is the protein of interest in the present investigation. The elevated levels of the enzyme aldose reductase are seen associated with diabetes nephropathy [25]. There are reports which states the action of the aldose reductase inhibitors, which could control the diabetes prognosis and further its complications [26,27]. This leads to a very important notion of the use of cancer drugs on diabetes. P.Vigneri et al. reported the use of 


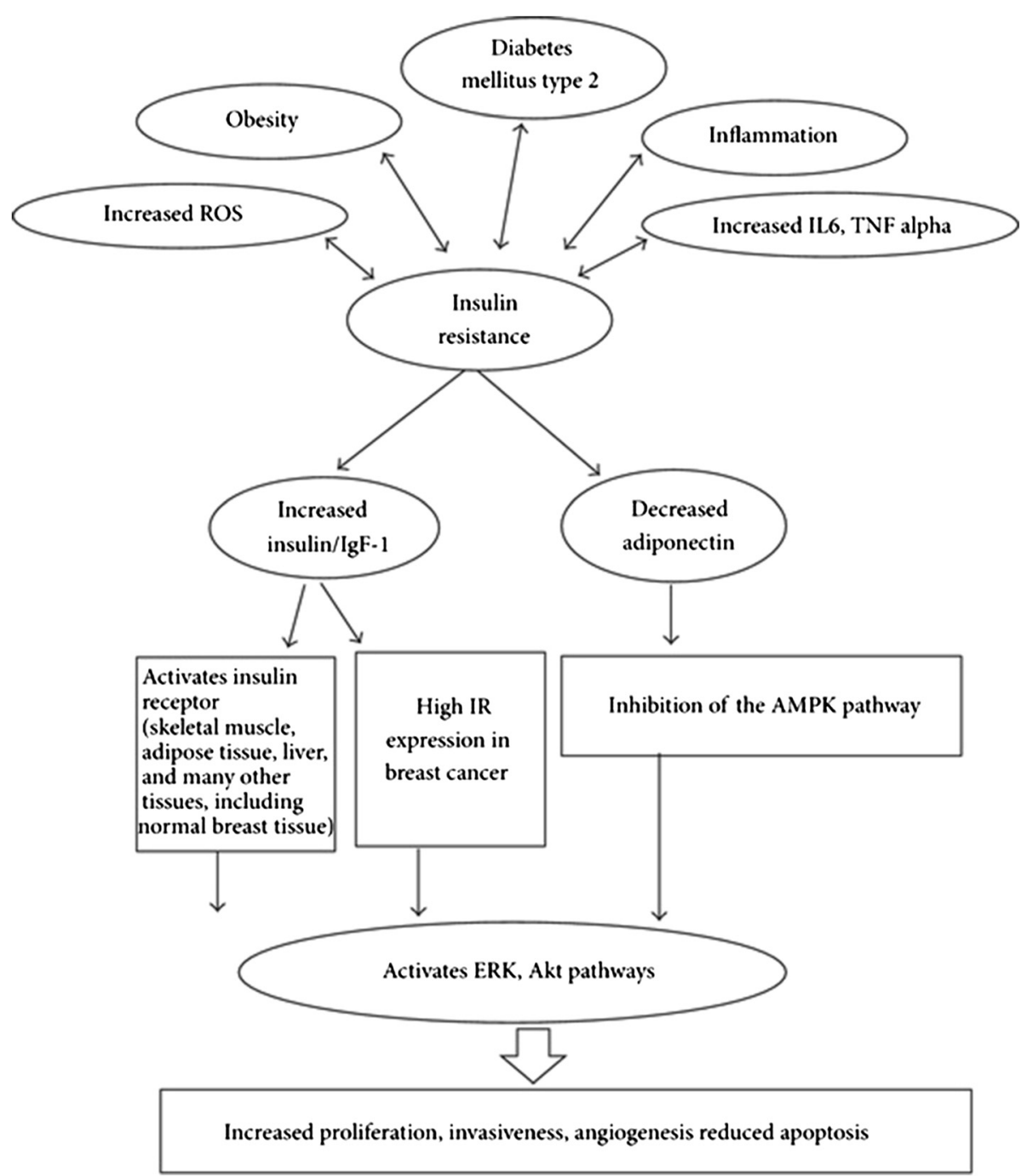

Figure 1 Pathophysiological link between Diabetes and Breast Cancer [22].

cancer drugs which favour the diabetes [28]. Hence, in the present article the breast cancer inhibitors are used to bind with the aldose reductase (1ADS).

Owing to this, the aim of the present article is to use the validated breast cancer inhibitors against the validated diabetic drug target protein via in silico analysis.
Materials and methods

Ligand preparation

The ligands used for the present study are validated breast cancer inhibitors [29]. Ten selected ligands were drawn using Marvin sketch, Figure 3 and subsequently imported into the Molegro.

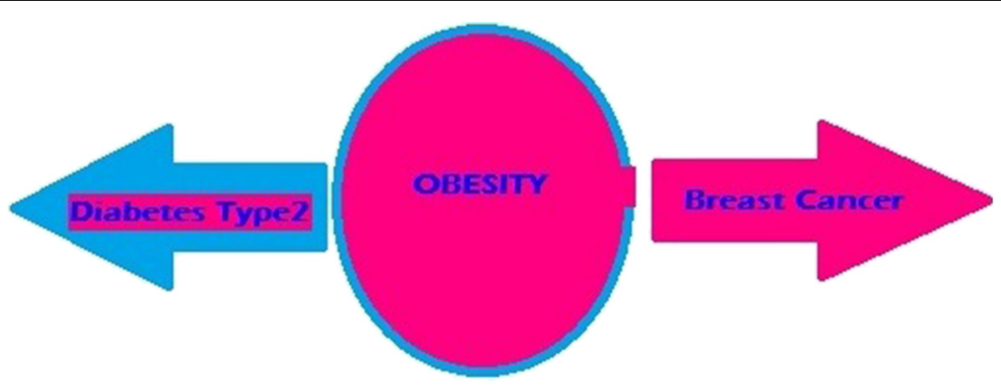

Figure 2 Depiction between obesity and breast cancer. 


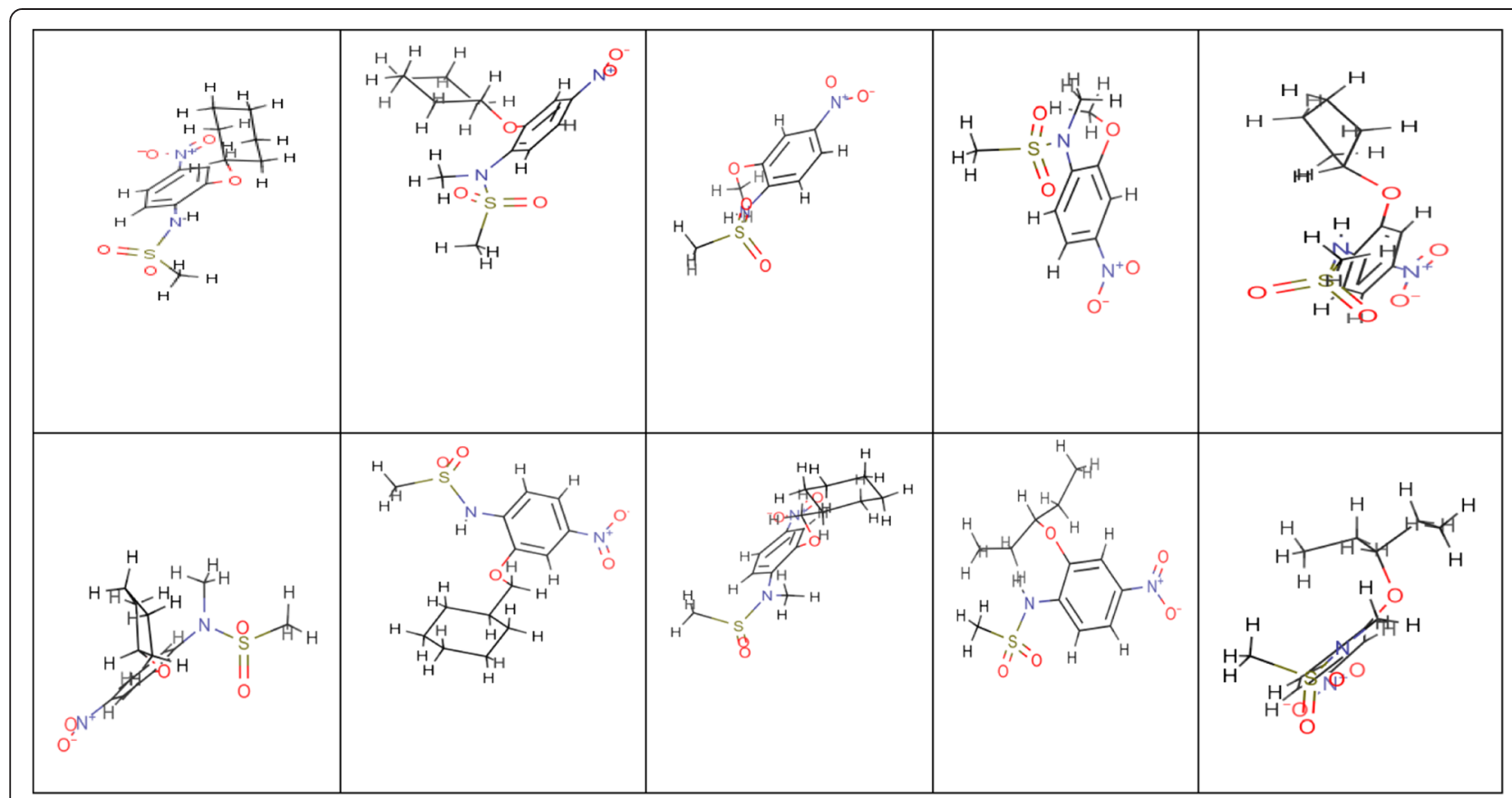

Figure 3 Breast cancer inhibitors.

\section{Protein selection and preparation}

The protein selected for the present study was 1ADS at the resolution $2.40 \AA$. The protein from the Protein Data Bank (PDB) was downloaded and imported into Molegro. The protein and the ligands were prepared assigning "always" to the given parameters.

\section{Docking}

The docking was done between the diabetes drug target and the breast cancer inhibitors on the Molegro docking wizard. The active sites of the protein were identified and the site with highest area and volume was selected for the docking, Figure 4.

\section{ヨ. Workspace: Unnamed \\ †. $\square$ Backbones [1] \\ $\square \square$ Cavities [5] \\ .... Cavity $\mathrm{Vol}=140.8]$ \\ $\square$ Cavity [Vol=49.664] \\ Cavity [Vol=37.888] \\ Cavity [Vol=10.752] \\ Cavity $[\mathrm{Vol}=10.24]$}

Figure 4 Volume of the active sites.

\section{Result}

The Molegro docking wizard revealed the following

The docking wizard generated 50 poses Figure 5. The highest dock score, -131.649 was seen with the inhibitor 1 . Hydrogen bond interactions were seen between the following amino acids Cys 298, Ser 210, Ser 159 and Asn 160 Figure 6.

\section{Discussion}

Diabetes mellitus is one of the major metabolic disorders, which is associated with several other complications like nephropathy, vascular diseases and hypertension [30]. Besides this, diabetes mellitus initiates tumour formation at most of the sites [31] associated with some cancers such as breast cancer [32] and prostate cancer [31]. Reports also indicate the association of diabetes with pancreatic cancer [33] Hence it is important to address the problem and to evaluate the relationship between them. The present article has successfully evaluated the use of the breast cancer drugs on 1ADS, a diabetic drug target. This is a novel approach through which both the morbidities can be reduced.

The 10 breast cancer inhibitors considered for the present study were proven to be effective against aromatase, an enzyme seen in the breast cancer patients [21]. As breast cancer is seen commonly in the post menopausal diabetic women it is ideal to use a drug which could treat both the aliments. Encouraged by this fact, the breast cancer drugs are used against diabetes drug target, unlike 


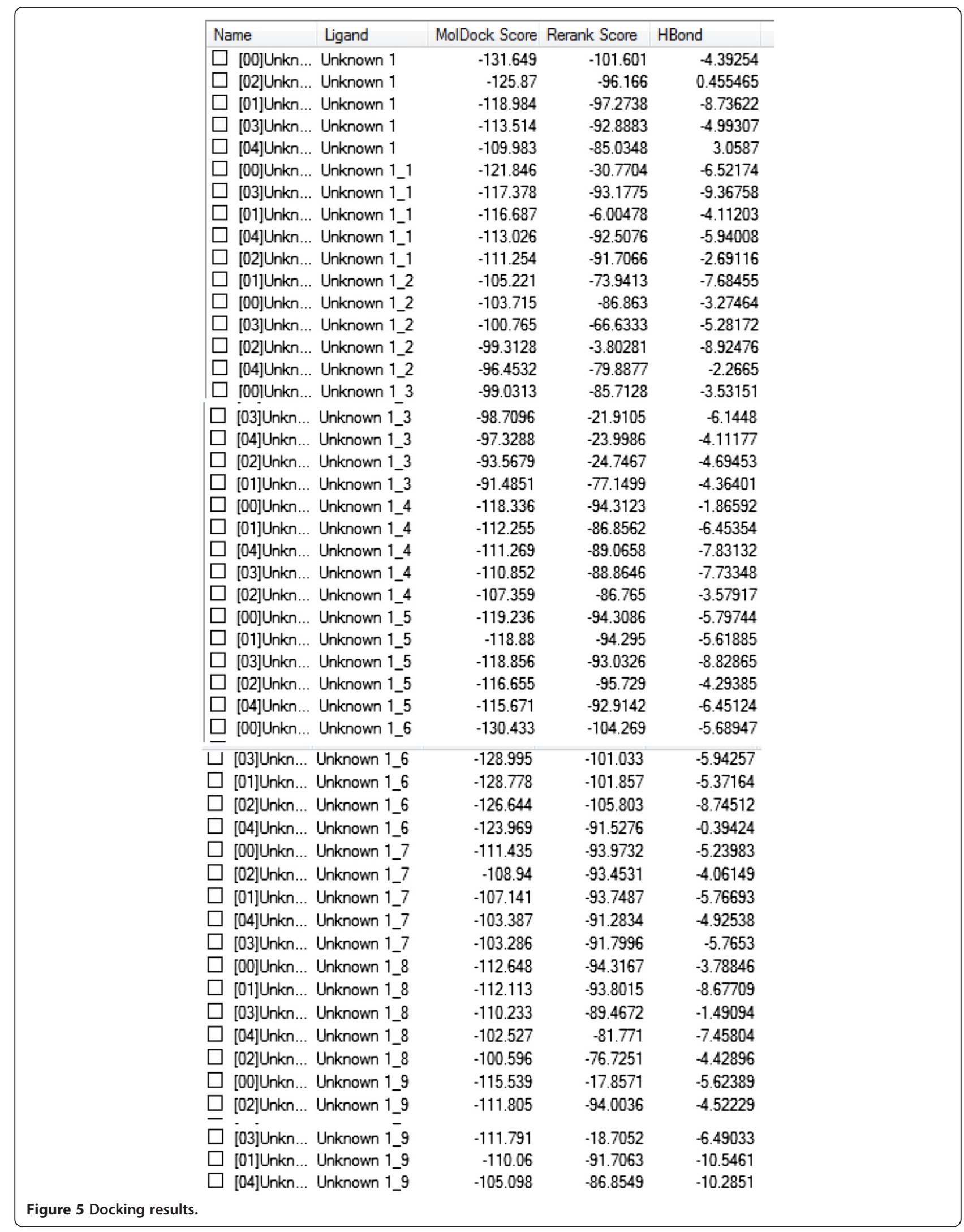




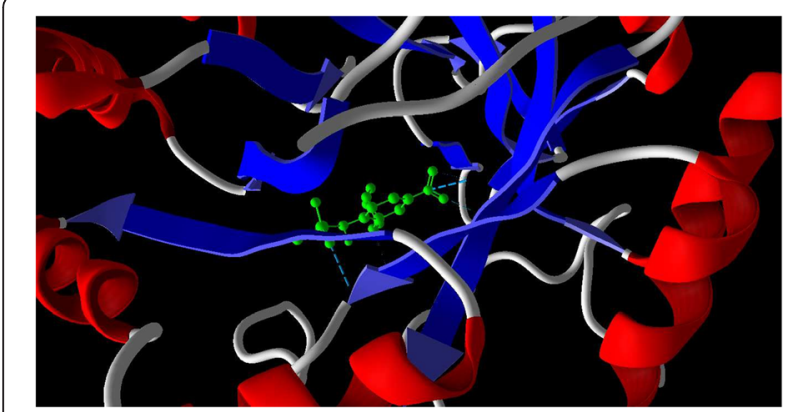

Figure 6 Docking result showing hydrogen bonds.

vice-a verse as previously described in this article. The results show a new dimension in the research for controlling both the diseases, hence can reduce both the morbidities.

\section{Conclusion}

Breast cancer and diabetes mellitus happen to be two of the major growing health concerns in the present day. Though not directly, diabetes mellitus is one of the causes of breast cancer incidences. It favours the growth of certain cancers like prostate cancer with a risk to other organs as well [28]. The present article successfully evaluates the role of breast cancer inhibitors on diabetes drug target. Given the best clinical attention, the associated relationship between both the diseases can be established, thus can cure both diseases.

\section{Competing interests}

The author declares that she has no competing interests.

Received: 10 February 2014 Accepted: 16 February 2015

Published online: 17 March 2015

\section{References}

1. Anderson GF, Chu E. Expanding priorities - confronting chronic disease in countries with low income. N Engl J Med. 2007;356:209-11.

2. Peairs KS, Barone BB, Snyder CF, Yeh HC, Stein KB, Derr RL, et al. Diabetes mellitus and breast cancer outcomes: a systematic review and meta- analysis. J Clin Oncol. 2011;29:40-6.

3. Calle EE, Murphy TK, Rodriguez C. Diabetes mellitus and pancreatic cancer mortality in a prospective cohort of United States adults. Cancer Causes Control. 1998;9:403-210.

4. Will JC, Vinicor F, Calle EE. Is diabetes mellitus associated with prostate cancer incidence and survival? Epidemiology. 1999;10:313-8.

5. Will JC, Galuska DA, Vinicor F, Calle EE. Colorectal cancer: another complication of diabetes mellitus? Am J Epidemiol. 1998;147:816-25.

6. Adami HO, McLaughlin J, Ekbom A. Cancer risk in patients with diabetes mellitus. Cancer Causes Control. 1991;2:307-14.

7. Chow WH, Gridley G, Nyren O. Risk of pancreatic cancer following diabetes mellitus: a nationwide cohort study in Sweden. J Natl Cancer Inst. 1995:87:930-1.

8. Wideroff L, Gridley G, Chow WH, Martha L. Cancer incidence in a population- based cohort of patients hospitalized with diabetes mellitus in Denmark. J Natl Cancer Inst. 1997:89:1360-5.

9. National Institute of Diabetes and Digestive and Kidney Diseases. National Diabetes Statistics Fact Sheet: General Information and National Estimates on Diabetes in the United States, 2007. Bethesda, MD: US Dept of Health and Human Services, National Institutes of Health; 2007.
10. American Cancer Society. Breast Cancer Facts and Figures 2007-2008. Atlanta, GA: American Cancer Society; 2008.

11. Giovannucci E. Insulin-like growth factor-I and binding protein-3 and risk of cancer. Horm Res. 1999;51 Suppl 3:34-41.

12. Ma J, Pollak MN, Giovannucci E. Prospective study of colorectal cancer risk in men and plasma levels of insulin-like growth factor (IGF)-1 and IGF-binding protein-3. J Natl Cancer Inst. 1999;91:620-5.

13. Grimberg A, Cohen P. Role of insulin-like growth factors and their binding proteins in growth control and carcinogenesis. J Cell Physiol. 2000;183:1-9.

14. Ernst C. Insulin-responsiveness of tumor growth. Arch Physiol Biochem. 2009;115:47-8

15. Coughlin SS, Calle EE, Teras LR, Petrelli J, Thun MJ. Diabetes mellitus as a predictor of cancer mortality in a large cohort of US adults. Am J Epidemiol. 2004;159:1160-7.

16. Chan JM, Stampfer MJ, Giovannucci E, Gann PH, Ma J, Wilkinson P, et al. Plasma insulin-like growth factor-1 and prostate cancer risk: a prospective study. Science. 1998;279:563-6.

17. Bohlke K, Cramer DW, Trichopoulos D. Insulin-like growth factor-1 in relation to premenopausal ductal carcinoma in situ of the breast. Epidemiology. 1998;9:570-3.

18. Shim M, Cohen P. IGFs and human cancer: implications regarding the risk of growth hormone therapy. Horm Res. 1999;51 Suppl 3:42-51.

19. Mantzoros CS, Tzonou A, Signorello LB. Insulin-like growth factor 1 in relation to prostate cancer and benign prostatic hyperplasia. $\mathrm{Br} J$ Cancer 1997;76:1115-8.

20. Giovannucci E, Rimm EB, Stampfer MJ. Diabetes mellitus and risk of prostate cancer (United States). Cancer Causes Control. 1998;9:3-9.

21. Vardhini SR. In silico evaluation for the potential naturally available drugs for breast cancer. J Recept Signal Transduct Res. 2014;34:174-9.

22. Hala A, Sami T. Azar: "Type 2 Diabetes Mellitus, Oral Diabetic Medications, Insulin Therapy, and Overall Breast Cancer Risk". ISRN Endocrinology. 2013;2013:1-8.

23. Masur K, Thévenod F, Znker KS. "Diabetes mellitus and breast cancer, "in Diabetes and Cancer. Epidemiological Evidence and Molecular Links. Front Diabetes. 2008;19:97-113.

24. Arcidiacono B, liritano S, Noceraetal A. "Insulin resistance And cancer risk: an overview of the pathogenetic mechanisms". Exp Diabetes Res. 2012;2012:1-12.

25. Shah VO, Dorin RI, Sun Y, Braun M, Zager PG. Aldose reductase gene expression is increased in diabetic nephropathy. J Clin Endocrinol Metab. 1997;82:2294-8.

26. Kador PF, Kinoshita JH, Norman E. Sharpless Aldose reductase inhibitors: a potential new class of agents for the pharmacological control of certain diabetic complications. J Med Chem. 1985;28:841-9.

27. Muthenna P, Suryanarayana P, Gunda SK, Petrash JM, Reddy GB. Inhibition of aldose reductase by dietary antioxidant curcumin: mechanism of inhibition, specificity and significance. FEBS Lett. 2009;583(22):3637-42.

28. Vigneri P, Frasca F, Sciacca L, Pandini G, Vigneri R. Diabetes and cancer. Endocr Relat Cancer. 2009;16:1103-23.

29. Su B, Tian R, Darby MV, Brueggemeier RW. Novel sulfonanilide analogs decrease aromatase activity in breast cancer cells: synthesis, biological evaluation, and ligand-based pharmacophore identification. J Med Chem. 2008;51:1126-35.

30. Sower JR, Epstein M. Diabetes mellitus and associated hypertension, vascular disease, and nephropathy. Hypertension. 1995;26:869-79.

31. Fall K, Garmo H, Gudbjörnsdottir S, Stattin P, Zethelius B. Diabetes mellitus and prostate cancer risk; a nationwide case-control study within PCBaSe Sweden. Cancer Epidemiol Biomarkers Prev. 2013;22:1102-9.

32. Liao S, Li J, Wei W, Wang L, Zhang Y, Li J, et al. Association between diabetes mellitus and breast cancer risk: a meta-analysis of the literature. Asian Pac J Cancer Prev. 2011;12:1061-5.

33. Morrison M. Pancreatic cancer and diabetes. Adv Exp Med Biol. 2012;771:229-39. 